\title{
Nuclearización de la infancia. La influencia nuclear en los cómics de Estados Unidos durante los años cuarenta y cincuenta
}

\section{Nuclearisation of Childhood. Nuclear Influence in American Comics during the Forties and Fifties}

\author{
Carlos Llorente Aguilera
}

Carlos Llorente Aguilera es licenciado en Psicología por la Universidad de La Laguna, máster en Ciencias de la Seguridad y Criminología por la Universidad Católica de Murcia y doctor en Historia Contemporánea por la Universidad Autónoma de Madrid. En la actualidad, se encuentra matriculado en el Programa de Doctorado en Periodismo de la Universidad Complutense de Madrid. Es autor de diversos artículos sobre historia y seguridad nuclear.

Fecha de recepción: 27 de julio de 2020

Fecha de aceptación definitiva: 30 de septiembre de 2020 


\title{
Resumen
}

El dominio de la energía nuclear por parte de Estados Unidos durante la II Guerra Mundial constituyó un hito sin parangón en la historia de la humanidad. Tras el fin de la contienda el elemento nuclear impregnó los más diversos e insospechados ámbitos de la vida. Una de las maneras en las que lo nuclear se abrió camino en el imaginario de la sociedad fue a través de los cómics infantiles que emplearon todas las variantes, reales o inventadas, atribuibles a la energía nuclear para lograr llegar a los corazones y mentes de los lectores más tiernos.

Palabras clave: Arma, Cómic, Estados Unidos, Nuclear, Superpoder.

\begin{abstract}
The dominance of nuclear energy by the United States during World War II was an unparalleled milestone in human history. After the end of the war, the nuclear element permeated the most diverse and unsuspected areas of life. One of the ways in which nuclear made its way into the imaginary of society was through children's comics that used all the variants, real or invented, attributable to nuclear energy to reach the hearts and minds of most tender readers.
\end{abstract}

Keywords: Comic, Nuclear, Superpower, United States, Weapon.

\section{Cita bibliográfica}

Llorente Aguilera, C. «Nuclearización de la infancia. La influencia nuclear en los cómics de Estados Unidos durante los años cuarenta y cincuenta», en CuCo, Cuadernos de cómic n. ${ }^{\circ} 15$ (2020), pp. 110-127. 


\section{Introducción}

El dominio de la energía nuclear por parte de Estados Unidos durante la II Guerra Mundial constituyó un trascendental hito de carácter científico, militar y económico dotado de unas proporciones no igualadas en el transcurso de la historia. La posibilidad de conseguir un arma de pequeñas proporciones dotada de un poder destructivo apenas concebible unos años antes y la sorprendente facilidad para conseguir transportarla al escenario de guerra y arrojarla sobre el enemigo en el corazón de sus ciudades, industrias, infraestructuras e instalaciones militares cambió de manera radical la manera de entender el arte de la guerra y la concepción que hasta ese momento se tenía del poderío militar como elemento de política, presión y defensa. La euforia vivida en el seno de la sociedad estadounidense de la época tras el conocimiento del bombardeo nuclear de Hiroshima y Nagasaki, y la subsiguiente y acelerada rendición de Japón, junto con la necesidad del país de enfrentarse a la nueva amenaza que significaba el creciente poder de la Unión Soviética, coadyuvaron al ascenso de la energía nuclear como panacea que podría propulsar a Estados Unidos en su nuevo papel de superpotencia. La magnitud de la empresa acometida para lograr el dominio sobre el proceso de fisión nuclear mediante el Proyecto Manhattan y la espectacularidad del rendimiento obtenido motivaron al gobierno estadounidense a perseverar en la tarea de impulsar, desarrollar y difundir la nueva tecnología, máxime cuando la Unión Soviética logró en 1949 el acceso a esta, con el fin de hacerse con un arsenal nuclear que asegurase al país su preponderancia en la escena internacional. No solo el gobierno del país se vio implicado en esta cruzada, ya que también la iniciativa privada se vio imbuida por el espíritu nuclear que impregnó los campos más variados de la existencia. Pronto, lo nuclear invadió todas las esferas de la vida pública y privada, consiguiendo hacerse un hueco en los ámbitos más insospechados, como pudieron ser la moda, la publicidad, los juguetes infantiles, las actividades de ocio o la hostelería. La infancia, como un objetivo más en esta campaña de promoción de las excelencias de lo nuclear, fue también abundantemente bombardeada, de forma que se emplearon a tal fin los medios más variopintos; entre ellos, la publicación de cómics fue uno de los más frecuentes y característicos durante el periodo de estudio de este artículo.

El cómic, como formato de historias cortas ilustradas, junto a las revistas de ciencia ficción, fue desde sus inicios un medio popular de entretenimiento y evasión con el que los más jóvenes podían acercarse a contenidos fantásticos o desconocidos que los alejaban momentáneamente de una realidad a menudo tediosa y falta de estimulación. Mención aparte hay que hacer a lo económico de este medio y a la sencillez de su acceso mediante la venta en los numerosos kioscos y librerías de la época, lo que, junto a la transmisión de estas publicaciones entre distintas generaciones de lectores, hicieron que este formato tuviera un gran impacto entre la infancia. Además, y como 
elemento fundamental en esta cuestión, es necesario destacar que lo nuclear estaba precisamente revestido del carácter novedoso y sensacional que podía hacer fortuna entre el público infantil y, con este propósito en mente, fue profusamente empleado por instituciones y editoriales para hacer más atractivas las publicaciones de la época.

Lo nuclear ya había sido tenido en cuenta por diversos autores como elemento protagonista, o investido de cierta relevancia, en varias obras precursoras de ciencia ficción que aparecieron a principios del siglo xx y que de una manera sorprendentemente acertada anticiparon algunos temas que posteriormente ilustrarían las páginas de los cómics. Obras como A Columbus of Space de Garrett P. Seviss, The Red Peril del Capitán Sterner St. Paul Meek, Solution Unsatisfactory de Robert Heinlein, Nerves de Lester del Rey, Deadline de Cleve Cartmill o The Paradise Crater de Philip Wylie, todas escritas antes de los ataques nucleares sobre Japón, emplean el elemento nuclear como eficaz herramienta que atrae y repele al mismo tiempo al lector, apenas mostrándole el poder encerrado en unas hipotéticas armas aún desconocidas para la mayoría de la humanidad.

Una vez la noticia del exitoso empleo de las bombas nucleares sobre Japón llegó a la población estadounidense y al resto del mundo, mediante la difusión de la noticia en periódicos y otros medios de comunicación, lo nuclear permeó con rapidez en la floreciente industria del cómic y sus efectos no tardaron en dejarse sentir en el nacimiento de nuevos géneros o en la ampliación de los ya existentes. La posterior carrera armamentística entre Estados Unidos y la Unión Soviética, fundamentalmente la relacionada con las armas nucleares y los correspondientes vectores de proyección, no hizo sino contribuir a la implantación del elemento nuclear en las revistas infantiles objeto de este artículo, lo que será oportunamente expuesto en las páginas siguientes.

\section{Objeto de estudio}

Como ha quedado ya esbozado en los párrafos anteriores, el objeto de estudio de la presente investigación se circunscribe a la manifestación del poder nuclear en la publicación de cómics dirigidos principalmente a la población infantil, aunque con un amplio consumo por parte de otros sectores de edad, en Estados Unidos y durante las décadas de los años cuarenta y cincuenta. El estudio se centra en este país debido a su especial significado en el desarrollo de la tecnología nuclear, ya que fue el pionero y la potencia dominante en todos sus aspectos ya sean bélicos o de uso pacífico. La utilización de este segmento temporal se justifica por la irrupción del elemento nuclear en el año 1945 y la sucesión de otros acontecimientos relevantes, tales como el inicio de la Guerra Fría entre Estados Unidos y la Unión Soviética, la obtención del poder nuclear por parte de esta última y el dominio de la tecnología de fusión nuclear aplicada a la guerra por parte de las dos superpotencias. A tal fin, se formula la hipótesis de que existe una poderosa influencia del elemento nuclear en la cultura en el ámbito 
espacial y temporal de este estudio, que se manifestó particularmente en la creación de un género nuevo dentro de las revistas infantiles denominadas genéricamente cómics, en el que ese factor nuclear es el absoluto protagonista.

\section{Estado de la cuestión}

La influencia del factor nuclear en los distintos medios de expresión cultural ha sido puesta de manifiesto por diversos autores con anterioridad, lo que da fe de la fuerza y del impacto que todo lo nuclear ha tenido en la vida, las costumbres, los conocimientos y las manifestaciones artísticas, tanto en Estados Unidos como en otros países. En un contexto amplio y general se encuentra la tesis de Andrew L. Bosse, presentada en 2013 y titulada Our Friend the Atom? The Truman Administration and the Campaign to Sell the Peaceful Atom, 1945-1949, en la que se analiza el trabajo de la Comisión para la Energía Atómica de Estados Unidos para hacer llegar a la población del país los aspectos pacíficos de la energía nuclear. Otra obra de gran enjundia es la tesis de Bradley J. Fest, presentada en 2013 y titulada The Apocalypse Archive: American Literature and the Nuclear Bomb, que expone el modo en el que la amenaza del conflicto nuclear ha modelado el desenvolvimiento de la literatura estadounidense en el siglo $\mathrm{xx}$, surgiendo como figura icónica la imagen del apocalipsis nuclear. Otro destacado autor que ha tratado esta cuestión de manera detallada y minuciosa es Paul Brians, con diversos artículos y estudios relativos a la aparición del elemento nuclear en obras de ciencia ficción. La tesis de 2017 de Joshua Samuel Scott Cornett Bombs, Bikinis, and Godzilla: America's Fear and Fascination of the Atomic Bomb as Evidenced Through Popular Media, 1946-1962, tiene también cabida en el presente estudio ya que se centra en el análisis de los cambios emocionales experimentados en la población de Estados Unidos durante los años cuarenta y cincuenta tras la irrupción del arma nuclear. Finalmente, y ya dentro del panorama nacional, se encuentra el interesante y excelentemente bien documentado artículo de José Joaquín Rodríguez Moreno «La energía atómica vista a través de la cultura popular estadounidense: una aproximación», publicado en 2011, en el que se hace una presentación general de la influencia de la energía nuclear en las expresiones culturales que surgieron en Estados Unidos desde el comienzo del siglo xx hasta el año 2011.

Ya en el ámbito del cómic, Matthew Pustz editó en 2012 la interesante obra Comic Books and American Cultural History, en la que analiza la importancia del cómic como testigo y reflejo de la historia dentro del marco de la cultura estadounidense. En 2014 vio la luz Comics as History, Comics as Literature. Roles of the Comic Book in Scholarship, Society, and Entertainment, de la mano de Annessa Ann Babic, en la que se desmenuza el papel jugado por los cómics en las sociedades como medio de enseñanza y entretenimiento. Kelly Elizabeth Shepherd analizó en su tesis de 2016 Tales of the Atom!: An Analysis of Nuclear Discourage in Early Marvel Comics la presencia del 
factor nuclear en los cómics de la compañía Marvel, muchos de cuyos protagonistas estaban dotados de superpoderes gracias a la acción de la energía nuclear. También es digno de mención, por su enlace entre el cómic y el elemento nuclear, el artículo de 2007 «Cartooning and Nuclear Power: From Industry Advertising to Activist Uprising and Beyond», de Leonard Rifas, en el que se muestra la influencia de la energía nuclear en el cómic, tanto desde el punto de vista publicitario como desde el propagandístico antinuclear.

Aunque la producción relativa al objeto de estudio es amplia, no existe una obra que atienda específicamente a la influencia del factor nuclear en el cómic estadounidense en las decisivas y fecundas décadas de los años cuarenta y cincuenta, por lo que queda abierta la puerta al desarrollo de este artículo como medio de remediar esa situación y de aportar al cuerpo de conocimientos global un nuevo elemento que describa y analice este tema, y que sirva, además, de referencia para futuros investigadores e interesados en la materia.

\section{Metodología}

La metodología empleada en la presente investigación es fundamentalmente heurística, y se basa en la búsqueda y recopilación de fuentes documentales relacionadas con el objeto de estudio en las que aparezca el elemento nuclear. Tras esta primera fase, y en este caso en particular, procede realizar un examen de las fuentes obtenidas, principalmente de manera cualitativa, de modo que se obtenga un lecho de datos suficiente como para proseguir con el estudio con garantías de éxito. Finalmente se analizarán los resultados a fin de estar en condiciones de corroborar la hipótesis formulada y de llegar al final del proceso a un conocimiento profundo del objeto de estudio.

En este sentido, se hará un uso intensivo de los amplios repositorios de cómics históricos Grand Comics Database, Comic Book + y Digital Comic Museum, que ofrecen importantes colecciones de material convenientemente digitalizadas y al alcance de los interesados en la cuestión. También la base de datos Health Physics Historical Instrumentation Museum Collection, de la Oak Ridge Associated Universities, es de gran utilidad para el propósito de esta investigación, ya que incluye diversa información sobre distintas manifestaciones culturales en las que el elemento nuclear cobra especial relevancia. Otros recursos de especial significación son los constituidos por los sitios web de The Atomic Heritage Foundation y de The National Museum of Nuclear Science and History. Finalmente se emplearán otros recursos de carácter general con el fin de contextualizar adecuadamente el relato, como pueden ser los sitios web The National Security Archive de The George Washington University y The Nuclear Weapon Archive en los que se encuentra abundante información, bien recopilada y adecuadamente contrastada. 


\section{Descubrimiento y euforia}

La imagen del hongo nuclear elevándose majestuosamente sobre el horizonte, alimentándose en su ascenso de las ruinas llameantes de un paisaje obliterado y extendiendo la destrucción a su alrededor, ya sea de forma inmediatamente constatable mediante el efecto de su onda expansiva o de las altas temperaturas generadas, o de una manera insidiosa mediante la lluvia radiactiva, forma parte del imaginario colectivo de la posguerra, de la Guerra Fría y de la actualidad, y su indudable poder de atracción ha sido puesto de manifiesto a través de una abundante producción cultural. Géneros tan dispares como el cine, la televisión, la literatura, la pintura o la música han sentido la influencia de lo nuclear en sus múltiples acepciones dejando constancia de la relevancia, del impacto, del significado y, sobre todo, de la trascendencia que esa imagen del hongo nuclear ha tenido, tiene y, presumiblemente, seguirá teniendo para las generaciones venideras.

Esta constatación del poder emanante de lo nuclear fue también aprovechada por las editoriales del pujante mundo del cómic de posguerra estadounidense para generar nuevos personajes y productos que pudieran ser acogidos con éxito entre el público infantil. El empleo de armas nucleares en los conflictos armados, los nuevos medios para hacer la guerra, la eclosión de la energía nuclear con fines de producción energética, el poder apenas intuido de la radiactividad y las dispares interpretaciones que de él se hicieron, la novedad, en definitiva, encerrada en el reciente dominio de la materia y de la energía y su dualidad encarnada en la posibilidad de alcanzar una destrucción inimaginable o de hacer el bien favoreciendo a la humanidad entera fueron poderosos acicates que hicieron bascular radicalmente la industria del cómic desde sus tradicionales posiciones hasta una nueva situación en la que lo nuclear adquiría un decidido protagonismo.

Una revisión exhaustiva de las publicaciones de la época permite encuadrar los esfuerzos realizados en tres categorías principales, a tenor del carácter protagonista del elemento nuclear. En primer lugar se encuentran los cómics protagonizados por héroes investidos de poderes excepcionales obtenidos gracias a la acción de la radiactividad, cuya principal actividad consiste en combatir el mal en sus múltiples encarnaciones. En una segunda categoría se pueden relacionar los cómics de carácter bélico, ambientados en diversos escenarios, en los que las armas nucleares tienen un papel destacado. En la tercera y última categoría pueden ubicarse los cómics de carácter educativo dedicados fundamentalmente a la difusión entre los más jóvenes de la energía nuclear, normalmente en su vertiente pacífica.

La influencia de lo nuclear en el mundo del cómic puede ser constatada además por el número de publicaciones en las que el protagonista era exclusivamente el factor nuclear o este aparecía en alguna de las historias incluidas en el interior de las publicaciones con el objeto de dotarlas de un mayor atractivo para los lectores debido a su 
carácter de materia de actualidad. En este sentido, y dentro del periodo de estudio, puede apreciarse la relevancia de este hecho en las siguientes cifras en las que se incluye año y número de apariciones del término atómico o atómica (atomic en inglés) o nuclear en los cómics editados (tabla 1 y gráfico 1, elaboración propia):

\begin{tabular}{|c|c|c|c|c|c|c|c|}
\hline Año & Atomic & Nuclear & Total & Año & Atomic & Nuclear & Total \\
\hline 1941 & 3 & - & 3 & 1951 & 40 & 2 & 42 \\
\hline 1942 & 3 & - & 3 & 1952 & 22 & 2 & 24 \\
\hline 1943 & 1 & - & 1 & 1953 & 57 & 4 & 61 \\
\hline 1944 & 2 & - & 2 & 1954 & 34 & 5 & 39 \\
\hline 1945 & 6 & - & 6 & 1955 & 33 & 1 & 34 \\
\hline 1946 & 63 & 1 & 64 & 1956 & 20 & 1 & 21 \\
\hline 1947 & 43 & 2 & 45 & 1957 & 35 & 4 & 39 \\
\hline 1948 & 20 & 2 & 22 & 1958 & 31 & 3 & 34 \\
\hline 1949 & 17 & 3 & 20 & 1959 & 33 & 10 & 43 \\
\hline 1950 & 19 & 1 & 20 & 1960 & 30 & 4 & 34 \\
\hline
\end{tabular}

FIG. 1. Apariciones de los términos atomic y nuclear en los cómics estadounidenses (1941-1960). Elaboración propia.

\section{EVOLUCIÓN PUBLICACIONES}

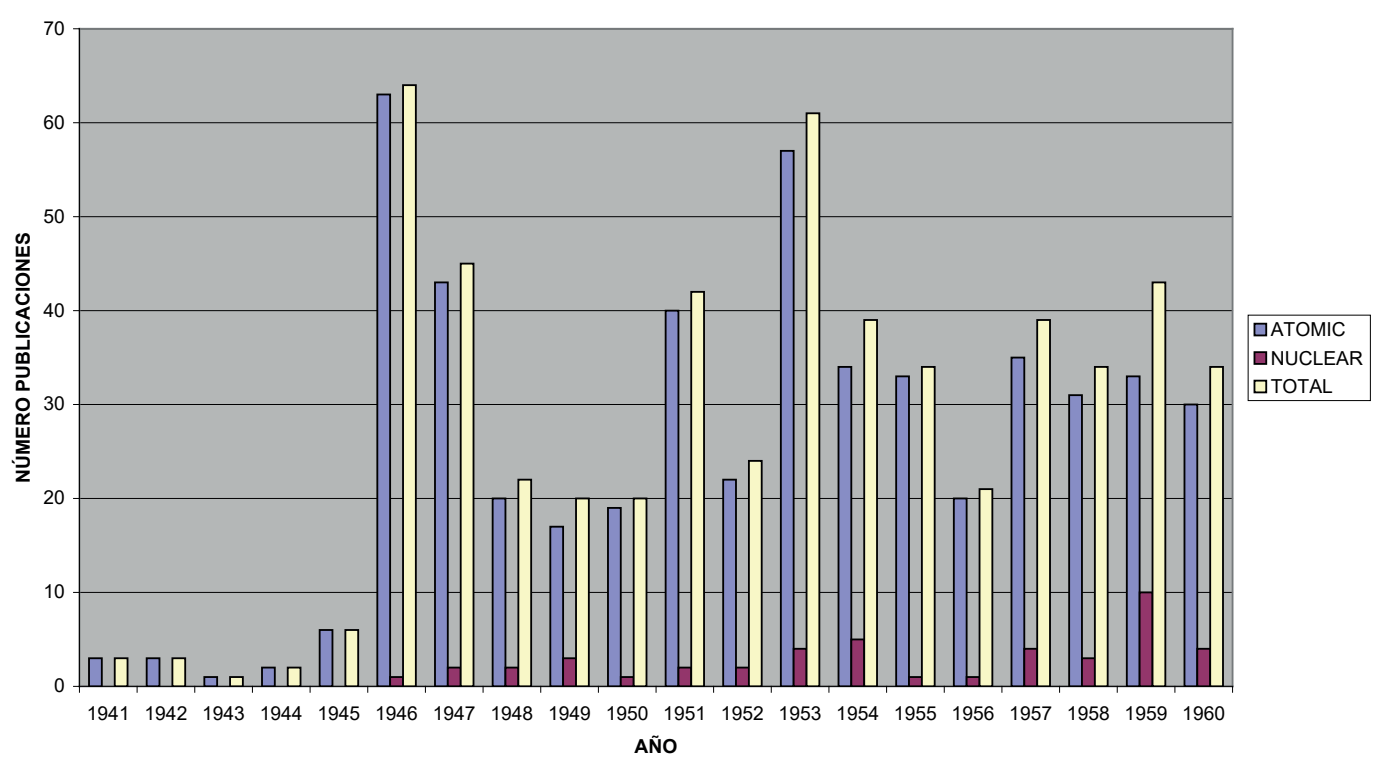

FIG. 2. Evolución del uso de los términos atomic y nuclear en los cómics estadounidenses (1941-1960). Elaboración propia. 
En las anteriores figuras puede verse con claridad el impacto del factor nuclear en los cómics a partir de 1945, con un mantenimiento estable superior a las veinte apariciones de los términos atomic y nuclear en los cómics publicados en el periodo de estudio. Como es natural, las cifras son consistentes con la realidad de la que eran reflejo, de forma que es importante el número de publicaciones en 1946 y 1947, una vez las noticias de los bombardeos nucleares en Japón se difundieron y arraigaron en la sociedad; en 1951, año en el que se produjo el primer ensayo con un arma de fusión por parte de Estados Unidos; y en 1953, año en el que la Unión Soviética alcanzó también el dominio sobre la fusión nuclear con fines bélicos.

\section{Superhéroes y radiación}

Respecto a la primera de las categorías se hace necesario mencionar como hito trascendente, en primer lugar, la aparición en 1938 de Superman, que supuso el nacimiento de un nuevo género en el que héroes dotados de poderes excepcionales luchaban contra el mal en sus variadas acepciones, a semejanza de los caballeros de los relatos medievales. Entre la plétora de héroes nacidos tras la estela de Superman se encuentra una rica multiplicidad de caracteres que obtenían sus superpoderes con el concurso de la radiación emitida de los elementos nucleares, tales como el uranio o el plutonio. El impacto de muchos de estos superhéroes queda patente en su longevidad en las páginas de los cómics y en su constante actualización para adaptarse al paso de los años y de las modas. No resulta banal la cuestión de que un proceso como la radiactividad, que mantiene una relación tan estrecha con la muerte y la destrucción, haya sido conceptualmente mutado de modo que finalmente haya sido aceptado beatíficamente por la mayoría del público como una especia de maná dotado de la capacidad de proporcionar poderes singulares y milagrosos a aquel que lo consume. No cabe duda de que este asunto es únicamente una pequeña muestra de la expuesta dualidad de la que está dotado todo lo que envuelve al poder nuclear y a la radiactividad, consistente en un superlativo aspecto destructivo hasta alcanzar proporciones apocalípticas y otro pacífico relacionado con aplicaciones percibidas como cuasi sobrenaturales.

El precursor de los superhéroes nacidos de la radiactividad fue el n. ${ }^{o} 19$ de Thrilling Comics, publicado por Better Publications en agosto de 1941, en el que se encuentra la historia de The American Crusader, en la que el protagonista, el profesor Masters, irrumpe inadvertidamente en un laboratorio en el que se estaba procediendo a "machacar un átomo», lo que acaba otorgándole superpoderes que empleará para la defensa de la democracia. Este estrambótico origen será compartido por la miríada de superhéroes que surgirán en los años venideros.

Otro cómic precursor en el empleo del factor nuclear fue Atomic Man, aparecido por primera vez en el número de noviembre de 1945 de la revista Headline Comics, edita- 
da por Prize. Este personaje recibía sus extraordinarios poderes tras sufrir una harto improbable serie de percances consecutivos, consistente en la ingesta de un vaso de agua pesada (ya de por sí radiactiva) en el que de manera accidental había caído una pequeña cantidad de uranio, con una posterior descarga de electricidad de alto voltaje que venía a completar la ecuación. Es notorio el hecho de que cualquiera de estos infortunios habría bastado para acabar con la vida de un ser humano pero, en esta particular y fantástica situación, tuvieron la inesperada consecuencia de transformar al protagonista en un ser nuevo, revitalizado con poderes sobrenaturales.

Otro superhéroe con una génesis similar al anterior fue Atoman, protagonista de dos cómics publicados a principios de 1946. En esta ocasión, Barry Dale, un científico inmerso en los trabajos del Proyecto Manhattan, era afectado por la radiación tras pasar varios años manipulando muestras de radio y uranio, adquiriendo los consabidos poderes ilimitados y convirtiéndose en Atoman. Valga como anécdota de la peculiar manera de entender la radiactividad por parte de las editoriales de estos cómics la explicación dada por el propio protagonista a los poderes recibidos, en la que exhibía como ejemplo a Marie Curie, descubridora del radio y el polonio, aunque bien sabido es que en el caso de la dos veces Premio Nobel la radiación no la convirtió en una superheroína, sino que acabó precozmente con su vida.

Un último exponente de esta categoría es Atomic Thunderbolt, con un solo número publicado en 1946 por Regor Company, y que pone al día la historia de Frankenstein, incluyendo en este caso a un científico dispuesto a trasmutar la materia mediante un experimento con elementos nucleares, que tiene la peculiaridad de transformar a un sujeto dejado de lado por la sociedad en un superhéroe dotado de una increíble fuerza.

Los poderosos efectos atribuidos a la radiación traspasaban todas las fronteras conocidas y muy pronto aparecieron nuevos superhéroes en el reino animal. El primero de ellos fue Atomic Mouse, publicado por Charlton Comics desde 1953 hasta 1963, cuyo protagonista es un ratón investido de superpoderes gracias a la oportuna ingesta de píldoras con un importante contenido de uranio, dedicando sus esfuerzos, a semejanza de sus iguales entre los seres humanos, a mantener el orden y la ley. Otro personaje de similares características es Atomic Rabbit, también de Charlton Comics y publicado entre 1955 y 1958, y en el que el personaje principal es un conejo dotado de poderes gracias a la ingesta de zanahorias con uranio. Un personaje similar fue Atomic Bunny, sucesor del anterior superconejo, igualmente de Charlton Comics y publicado entre 1958 y 1959. Atom the Cat, publicado por la misma editorial entre 1957 y 1959, trata de un gato dotado de superpoderes, como es natural, tras ser irradiado por la acción de un reactor nuclear en el que unos científicos, según explican en una de las viñetas, no estaban únicamente procediendo a la fisión de los núcleos de elementos tales como el uranio y el plutonio, sino que habían traspasado las fronteras de la ciencia al «pulverizarlos». 
Además de los personajes citados, muchos otros superhéroes se vieron envueltos en situaciones en las que debían enfrentarse a oponentes que pretendían emplear armas nucleares para llevar a cabo sus fechorías, como fue el caso del Capitán América en "Mistery of the Atomic Boomerang», publicado por Marvel en 1945 en el n. ${ }^{\circ} 51$ de Captain America, Robin en «The Stolen Atom Bomb», publicado por DC en 1947 en el n. 69 de Star Spangled Comics, Superman en «A Superman of Doom!», publicado por DC en 1948 en el n. ${ }^{\circ} 124$ de Action Comics, la Familia Marvel en «The Marvel Family Fights the Atomic Crimes!», publicado por Fawcett en 1952 en el n. ${ }^{\circ} 76$ de The Marvel Family o Batman en «Menace of the Atomic Man!» publicado por DC en 1960 en el n. 280 de Detective Comics.

\section{El arma nuclear}

En la segunda de las categorías expuestas se encuentran los cómics en los que las armas nucleares adquieren el papel protagonista, con dos vertientes, la bélica y la delictiva. Respecto a la primera de estas vertientes, ya antes de la irrupción del arma nuclear en la II Guerra Mundial los cómics estadounidenses contenían historias ambientadas en la contienda, en las que se trasladaban las victorias y hazañas de las fuerzas armadas de Estados Unidos a los más pequeños, con exponentes tales como Air Fighters Comics, publicado por Hillman entre 1941 y 1945, Captain Aero Comics, publicado por Holyoke entre 1942 y 1943, o The American Airforces, publicado por Magazine Enterprises entre 1944 y 1945. Respecto al elemento nuclear, y al igual que ocurriera con los relatos de ciencia ficción expuestos en el apartado correspondiente a la introducción, los cómics adelantaron a sus lectores lo que estaba por llegar y en diversas publicaciones se encuentran muestras que anticipan las armas nucleares y la radiactividad. Un ejemplo de esto es el n. ${ }^{\circ} 11$ de Target Comics, publicado por Novelty Press en una fecha tan temprana como diciembre de 1940, en el que se incluye la historia de Lucky Byrd titulada "The Atomic Bomb», en la que los enemigos de Estados Unidos, denominados Zorians, anuncian que van a llevar a cabo diversos ensayos con bombas atómicas que finalmente resultan ser un engaño. La capacidad de anticipación del poder de lo atómico resulta clara en esta historia, ya que la esencia del argumento era la explosión de una pequeña bomba con un inimaginable poder destructivo, algo que en 1945 pasaría tristemente de la ficción a la realidad.

Otra historia con poder de anticipación fue el relato de Wings Wendall, aparecido en el n. ${ }^{\circ} 31$ de Smash Comics, publicado por Quality en febrero de 1942, tan solo dos meses después del ataque japonés a Pearl Harbor, en el que se detalla el plan de un enemigo imaginario de Estados Unidos, «la amenaza roja», personificado en individuos con rasgos orientales que pretenden destruir Washington mediante el empleo de una bomba atómica. Otro ejemplo lo constituye la historia de Jun-Gal, la diosa blanca de la tribu de los Tagomas, en África, aparecida en el n. 2 de Blazing Comics, publicado por Rewl Publications Inc. en julio de 1944, en la que se relata la incursión 
de las tropas alemanas para hacerse con una cantidad del elemento radio, necesario para la fabricación de un arma denominada «alterador atómico». Este relato de fantasía no estaba demasiado alejado de la realidad, ya que la Alemania nazi acumuló grandes cantidades de uranio, que fueron descubiertas por las tropas estadounidenses en 1944, y que estaban destinadas al primitivo programa nuclear alemán.

Tras el fin de la contienda mundial la presencia de lo nuclear en los cómics se hace arrolladora. La pionera en este aspecto es The Secret Voice, publicada por American Features Syndicate en 1945, en la que se ofrece a los lectores una curiosa historia sobre un microscópico insecto cuya principal ocupación es la de enviar las ideas apropiadas a la mente de los líderes y combatientes de Estados Unidos para que puedan triunfar en la II Guerra Mundial. Una de esas ideas es, como no podía ser de otra manera, la que ayuda a los científicos a finalizar sus trabajos sobre la bomba nuclear que acabará con la rendición de Japón.

Ya en 1952, apareció en los kioscos Atomic War, publicado por Ace Comics hasta 1953, coincidiendo con el conflicto armado en la península coreana, en el que se presentaba un enfrentamiento entre los bloques liderados por Estados Unidos y la Unión Soviética con el crudo empleo de armas nucleares. Estos cómics llevaban un aclarador antetítulo que rezaba «Solo una América fuerte puede prevenir la guerra atómica», complementado por la publicidad de su interior en la que se animaba a los lectores a la compra de Bonos de Defensa del Gobierno de Estados Unidos como forma de colaborar con el esfuerzo de guerra. Otra publicación es Atom-Age Combat, publicada por St. John Publications entre 1952 y 1953, en la que se presentaban diversas historias con el mismo enfrentamiento nuclear entre bloques. Atomic Attack, de Youthful Magazines y publicado en 1953, mostraba la misma ambientación en la que no faltaban las ubicuas y espectaculares explosiones nucleares.

Commander Battle and the Atomic Sub, publicado por American Comics Group en 1954, se apartaba de la línea establecida en los cómics anteriormente expuestos y la acción era protagonizada por un submarino nuclear, cuyos adversarios eran seres dotados de superpoderes, que además contaban con platillos volantes para su propósito de dominar la Tierra. En este caso, la novedad del cómic consiste en la inclusión de la recientemente descubierta propulsión nuclear, ya que el primer submarino nuclear de Estados Unidos, el Nautilus, había sido botado precisamente ese año de 1954.

En cuanto a la vertiente protagonizada por delincuentes que emplean el elemento nuclear para dar mayor realce a sus fechorías, en el verano de 1946 salió a la venta el n. ${ }^{\circ} 4$ de Atomic Comics en el que se incluye la historia «The Atomic Blondshell», protagonizada por la audaz reportera Lucky Wings, que se encarga de desbaratar un complot del hampa en el que tiene un papel destacado la energía nuclear. En diciembre de 1946 Quality Comic Group publicó el n. ${ }^{\circ} 68$ de Smash Comics con una historia 
en la que el protagonismo lo tienen unas armas nucleares en forma de dados. En junio de 1947 salió a la venta el n. ${ }^{\circ} 22$ de America's Best Comics, publicado por Pines, en el que se podía encontrar la historia de The Black Terror, un justiciero que se encargaba de desbaratar los planes de John Reece, un delincuente que pretendía construir una plataforma volante con capacidad de arrojar bombas nucleares sobre las principales ciudades de la Tierra. También la compañía Disney sintió la influencia de lo nuclear, y publicó en 1947 una breve historia denominada Donald's Duck Atom Bomb, que podía encontrarse en el interior de las cajas de cereales Cheerios, y en la que el Pato Donald inventa una bomba nuclear cuyo principal efecto es el de causar alopecia entre los personajes cercanos, y cuya fórmula es robada por un científico extranjero que pretende huir a su país con el secreto entre sus manos.

\section{La vertiente educativa}

Ya en relación a la tercera de las categorías expuestas, la referente a los cómics editados con propósitos educativos y de difusión de la ciencia nuclear, es necesario expresar que estas publicaciones fueron en muchas ocasiones patrocinadas por la industria nuclear o desde el gobierno de Estados Unidos a fin de publicitar la vertiente pacífica de la energía nuclear y separarla convenientemente de su aplicación bélica. La publicación pionera en esta categoría fue Science Comics, de Ace Magazines, cuyo n. ${ }^{\circ} 1$ vio la luz en enero de 1946, y que trata, a través de historias cortas, diversas cuestiones científicas. La primera de ellas es la dedicada a los orígenes de las primeras bombas nucleares. En marzo de 1946 apareció el n. ${ }^{\circ} 1$ de Marvels of Science, de la mano de Charlton Publications, en la que se incluían diversos relatos de distinto calado, destacando la historia de la evasión del científico danés Niels Bohr, pionero en las investigaciones que derivaron en la fabricación del arma nuclear, para escapar de las garras del nazismo en Europa. También en marzo de 1946 vio la luz el n. ${ }^{\circ} 29$ de Real Life Comics, publicado por Nedor Publishing Company, en el que aparece una historia similar a la anterior en la que se relatan los orígenes del arma nuclear y la implicación de los científicos huidos de Europa, entre los que se encontraba el mencionado Niels Bohr. En abril de este mismo año apareció el n. ${ }^{\circ} 2$ de la revista Marvels of Science, editada por Charlton Publications, que dedicaba varias de sus páginas a la energía nuclear y a sus posibles aplicaciones en el futuro. Igualmente en 1946 fue publicada por George W. Dougherty Publishing Company Future World, cuyo número de verano incluía una amena y coloridamente ilustrada explicación sobre el funcionamiento de la energía nuclear.

En el segmento de publicaciones enteramente dedicadas a la cuestión nuclear se encuentra Adventures inside the Atom, editada en 1948 por la General Electric Company a instancias de la Comisión para la Energía Atómica de Estados Unidos, en la que se hacía un recorrido histórico de los hitos que condujeron al descubrimiento de la energía nuclear. La separación de los dos aspectos de la energía nuclear es clara en este 
cómic, ya que no se hace mención a las armas nucleares ni hay llamativas ilustraciones protagonizadas por el hongo nuclear, y se pasa además de puntillas por el desarrollo del Proyecto Manhattan.

Otro cómic dotado de singular relevancia en esta categoría es Dagwood Splits the Atom, publicado por la agencia de prensa King Features Syndicate en 1949, en la que se desentrañan y muestran a los más jóvenes los misterios alojados en el interior del átomo mediante la combinación de viñetas y textos complementarios. Además, cuenta con la participación de relevantes personajes de la compañía, tales como Popeye el marino y el mago Mandrake. La publicación incluye un cuestionario para evaluar de manera amena los conocimientos adquiridos tras la lectura y, al igual que ocurría en Adventures inside the Atom, las referencias al aspecto bélico de la energía nuclear no aparecen, a excepción de una oblicua mención en las páginas veinticinco y veintiséis. Este cómic fue apadrinado por el General Leslie Groves, director del Proyecto Manhattan, que escribió el prólogo, en el cual mostraba su esperanza de que los jóvenes lectores fueran estimulados por la lectura de la publicación para que perseveraran por la senda del conocimiento en pos de la paz mundial.

Otro cómic más editado con fines educacionales fue el número de septiembre de 1953 de Picture Parade, en el que se relatan las aventuras atómicas de Andy, un niño, y su perro Spot, habitantes de Middletown, en el Estado de Nevada, tan solo a treinta millas del lugar en el que se llevan a cabo los ensayos nucleares. Al comienzo de la historia el perro se interna en la zona en la que se va a ensayar un arma nuclear y el niño vuelve a casa desconsolado y pensando en la maligna naturaleza de los átomos que acabará con la vida de su mascota. Varias visitas del niño acompañado por su padre al hospital en el que su madre está recibiendo radioterapia y a diversos centros de investigación sirven para dar paso a sucesivas explicaciones sobre los beneficios de la energía nuclear que muestran su vertiente pacífica (para tranquilidad de los lectores es conveniente indicar que el perro aparece sano y salvo al final de la historia). En este cómic se incluían además un test y un crucigrama para probar los conocimientos de los jóvenes lectores sobre la energía nuclear.

Ya en 1955 se reeditó Adventures inside the Atom, con el título simplificado de Inside the Atom, de modo que se procedía a su actualización y a una mejora de las ilustraciones. Dos años después, en 1957 M. Philip Copp. publicó The Atomic Revolution, un cómic con un carácter realmente innovador, debido a la extraordinaria calidad de sus viñetas y la fundamentación científica de los textos, en el que se detalla la historia de la energía nuclear y se exponen sus aplicaciones de modo instructivo. Finalmente, en The Mighty Atom, cómic publicado en 1959 por Whitman en 1959, se encuentra detallada la historia de la electricidad desde sus comienzos hasta la llegada de la energía nuclear, contándose para este menester con la colaboración de la mascota publicitaria de la industria eléctrica estadounidense de la época, Reddy Kilowatt. 
En este apartado dedicado a los cómics de carácter didáctico es necesario mencionar aquellas publicaciones dedicadas a concienciar y formar a la población civil sobre el modo en el que debía reaccionar en caso de ataques con armas nucleares, una posibilidad real una vez la Unión Soviética adquirió la capacidad de proyectar sus armas nucleares sobre territorio estadounidense. De este modo, en 1951 apareció If an A-Bomb Falls, de la mano del Departamento de Defensa Civil del Estado de Delaware, que recogía en un formato fácilmente accesible a los más jóvenes los consejos básicos necesarios para sobrevivir a un ataque nuclear. Del mismo tenor era el cómic Bert the Turtle Says Duck and Cover, cuyo principal personaje era la tortuga Bert, protagonista además de la campaña de gran difusión en formato de dibujos animados «Duck and Cover» de 1951 y 1952, de la Administración Federal de Defensa Civil, que tenía el objetivo de mostrar a los niños la manera de proceder en caso de ataque nuclear. Una obra más en esta categoría la constituye The H-Bomb and You, publicado en esta ocasión en 1955 por la Agencia de Defensa Civil del Estado de Maryland, en la que en un formato similar al de If an A-Bomb Falls se alerta a los más jóvenes sobre la manera de proceder, teniendo en este caso en cuenta la mayor potencia de las armas termonucleares o de fusión. Por último en esta categoría, en 1957 y de la mano de Graphic Information Service, vio la luz Operation Survival, un cómic orientado a mostrar a los más jóvenes los fundamentos de la defensa civil en caso de desastres, en el que tiene un papel destacado la protección ante ataques nucleares, y en el que se incluyen, como en otras publicaciones similares, un cuestionario y un crucigrama para evaluar los conocimientos adquiridos tras la lectura.

Finalmente, $\mathrm{y}$ en un formato que puede encontrarse a medio camino entre el cómic y el cuento, apareció en 1956 de la mano de Walt Disney Our Friend the Atom, magna publicación en la que se detalla la historia de la energía hasta llegar a la aparición y desarrollo de la nuclear, exponiéndose las múltiples aplicaciones pacíficas de esta última y el beneficio que suponían para el bienestar de la humanidad. Esta obra contó con la colaboración del físico Heinz Haber, que se ocupó de los textos, y de una veintena de ilustradores de la factoría Disney, que dejaron su singular impronta en los dibujos que jalonan la publicación.

\section{Conclusiones}

Tras el estudio realizado, parece oportuno ofrecer las siguientes conclusiones, que vienen en última instancia a corroborar la hipótesis que fue inicialmente formulada. Tal y como ha quedado expuesto en los apartados precedentes, la violenta irrupción de la energía nuclear en el epílogo de la II Guerra Mundial en forma de ataque decisivo sobre Hiroshima y Nagasaki supuso un elemento de inspiración e influencia en el mundo de la cultura de posguerra y años sucesivos, y esto se dejó sentir de manera inequívoca y palpable en los cómics en los que la presencia de ese factor era prácticamente nula hasta ese momento. 
La aparición de tres nuevos géneros de publicaciones centrados principalmente en los superhéroes emergidos de la radiación, en el conflicto nuclear y en la difusión tanto de los beneficios de la energía nuclear como de la manera de protegerse contra su uso perverso (siempre por parte del enemigo) fue la principal manifestación de esa influencia nuclear que adquirió tintes de protagonismo absoluto en publicaciones monográficas y que además alcanzó a personajes, editoriales y revistas ya consolidadas que se impregnaron del espíritu de la época para atraer a un público más numeroso hasta sus páginas. Este espíritu alcanzó tales cotas de paroxismo que tuvo incluso el extraño efecto de obviar, en el caso de los superhéroes expuestos en este estudio, los perniciosos efectos provenientes de la radiactividad transformándolos en fuente inagotable de poder y de salud, lo que decididamente contribuyó a imprimir un marcado carácter a estos personajes que los hizo más atractivos a ojos de los ávidos consumidores infantiles. Es además relevante la implicación de instituciones públicas y privadas en la promoción de la vertiente educativa de los beneficios de la energía nuclear precisamente en dos momentos clave de este periodo, situados uno en los comienzos de andadura de la incipiente industria nuclear y en la necesidad de hacer llegar a la sociedad la importancia de este nuevo tipo de energía, y el otro en el apremio por concienciar de manera eficaz e indeleble a la población de las medidas que debía adoptar para protegerse de un eventual ataque nuclear una vez se produjo la eclosión del enfrentamiento larvado entre Estados Unidos y la Unión Soviética y la posibilidad de un conflicto nuclear generalizado pareció cierta e inevitable.

Puede afirmarse, además, que esta influencia se mantuvo estable durante el periodo de estudio, sin mostrar síntomas de agotamiento, y generando nuevos personajes y situaciones en las que el elemento nuclear seguía estando presente de una manera notable, lo que viene a constatar la decidida importancia y la persistente vigencia del mismo. En definitiva, para finalizar con esta disertación y a tenor de los datos que anteceden, resulta oportuno concluir que la aparición de la energía nuclear en sus acepciones bélica y pacífica, y su consiguiente irrupción en la vida social influyeron de manera significativa en las manifestaciones culturales del periodo de estudio, lo que es especialmente relevante en el objeto de atención del presente artículo, los cómics dirigidos al público infantil. 


\section{BibLiografíA}

BABIc, V. A. (coord.). Comics as History, Comics as Literature. Roles of the Comic Book in Scholarship, Society, and Entertainment. Plymouth, Fairleigh Dickinson University Press, 2014.

Bosse, A. L. Our Friend the Atom? The Truman Administration and the Campaign to Sell the Peaceful Atom, 1945-1949 [tesis doctoral]. Chico, California State University, 2013. Disponible en: http://csuchico-dspace.calstate.edu/handle/10211.3/10211.4 611

Fest, B. J. The Apocalypse Archive: American Literature and the Nuclear Bomb [tesis doctoral].Pittsburgh,University of Pittsburgh, 2013. Disponible en: http://d-scholarship.pitt.edu/18329/

Pustz, M. (coord.). Comic Books and American Cultural History. Nueva York. Continuum International Publishing Group, 2012.

Rifas, L. «Cartooning and Nuclear Power: From Industry Advertising to Activist Uprising and Beyond", en PS: Political Science E Politics n. ${ }^{\circ}$ 2, 40, online, Cambridge University Press, 2007, pp. 255-260.

Rodríguez Moreno, J. J. «La energía atómica vista a través de la cultura popular estadounidense: una aproximación», en Investigaciones Históricas n. ${ }^{\circ}$ 31, Universidad de Valladolid, 2011, pp. 165-200.

Scott Cornett, J. S. Bombs, Bikinis, and Godzilla: America's Fear and Fascination of the Atomic Bomb as Evidenced Through Popular Media, 1946-1962 [tesis doctoral]. Richmond, Eastern Kentucky University, 2017. Disponible en: https://encompass. eku.edu/etd/480/

Shepherd, K. E. Tales of the Atom!: An Analysis of Nuclear Discourage in Early Marvel Comics [tesis doctoral]. Columbia, University of South Carolina, 2016. Disponible en: https://scholarcommons.sc.edu/senior theses/80/

\section{RECURSOS WEB}

Comic Book +. Disponible en: https://comicbookplus.com/

Digital Comic Museum. Disponible en: https://digitalcomicmuseum.com/ 
Grand Comics Database. Disponible en: https://www.comics.org/

Nota: Todos los cómics citados en el presente artículo pueden ser consultados en los anteriores sitios web.

Health Physics Historical Instrumentation Museum Collection. Disponible en: https://www.orau.org/health-physics/museum.html

The Atomic Heritage Foundation. Disponible en: https://www.atomicheritage. org/

The National Museum of Nuclear Science and History. Disponible en: https:// www.nuclearmuseum.org/

The National Security Archive. Disponible en: https://nsarchive.gwu.edu/ The Nuclear Weapon Archive. Disponible en: https://nuclearweaponarchive.org/ The Web Site of Professor Paul Brians. Disponible en: https://brians.wsu.edu/ 